\title{
Association of the Hermansky-Pudlak syndrome type 4 (HPS4) gene variants with cognitive function in patients with schizophrenia and healthy subjects
}

Go Kuratomi ${ }^{1}$, Atsushi Saito ${ }^{1}$, Yuji Ozeki ${ }^{2}$, Takashi Watanabe ${ }^{2}$, Kumiko Fujii ${ }^{2}$, Kazutaka Shimoda ${ }^{2}$, Toshihiko Inukai ${ }^{3}$, Harunobu Mori ${ }^{4}$, Kenichi Ohmori ${ }^{5}$ and Kazufumi Akiyama ${ }^{{ }^{*}}$

\begin{abstract}
Background: The Hermansky-Pudlak Syndrome Type 4 (HPS4) gene, which encodes a subunit protein of the biogenesis of lysosome-related organelles complex (BLOC)-3, which is involved in late endosomal trafficking, is associated with schizophrenia; however, its clinical relevance in schizophrenia remains unknown. The purpose of the present study was to investigate whether HPS4 is associated with cognitive functions in patients with schizophrenia and healthy controls and with the clinical profiles of patients with schizophrenia.

Methods: We investigated the association of variants of HPS4 with clinical symptoms and cognitive function in Japanese patients with schizophrenia $(n=240)$ and age-matched healthy control subjects $(n=240)$ with single nucleotide polymorphisms (SNP)- or haplotype-based linear regression. We analyzed five tagging SNPs (rs4822724, rs61276843, rs9608491, rs713998, and rs2014410) of HPS4 and 2-5 locus haplotypes of these five SNPs. The cognitive functions of patients and healthy subjects were evaluated with the Brief Assessment of Cognition in Schizophrenia, Japanese-language version, and the patients were assessed for their symptomatology with the Positive and Negative Symptom Scale (PANSS).

Results: In patients with schizophrenia, rs 713998 was significantly associated with executive function under the dominant genetic model $(P=0.0073)$. In healthy subjects, there was a significant association between working memory and two individual SNPs under the recessive model (rs9608491: $P=0.001$; rs713998: $P=0.0065$ ) and two haplotypes (rs9608491713998: $P=0.0025$; rs61276843-9608491-713998: $P=0.0064$ ). No significant association was found between HPS4 SNPs and PANSS scores or premorbid IQ, as measured by the Japanese version of the National Adult Reading Test.

Conclusions: These findings suggested the involvement of HPS4 in the working memory of healthy subjects and in the executive function deficits in schizophrenia.
\end{abstract}

Keywords: HPS4, Cognition, Working memory, Executive function, BACS, rs9608491, rs713998

\section{Background}

Cognitive impairments are a core feature of schizophrenia and a major determinant of functional outcome [1]. Cognitive domains that are particularly impaired in patients with chronic schizophrenia are verbal memory, working memory, motor speed, verbal fluency, attention, and executive function $[2,3]$. These cognitive domains, specifically working memory and executive function, are

\footnotetext{
* Correspondence: kazufuak@dokkyomed.ac.jp

'Department of Biological Psychiatry and Neuroscience, Dokkyo Medical University School of Medicine, 880 Kitakobayashi, Mibu, Tochigi 3210293, Japan Full list of author information is available at the end of the article
}

heritable traits that have a potential association with genetic variants $[4,5]$. Therefore, these traits are useful as an intermediate phenotype to investigate the functional association of susceptibility genes for schizophrenia [6,7].

$22 \mathrm{q} 12$ is one of the regions that show high logarithm of the odds ratio scores in schizophrenia [8-10]. The Hermansky-Pudlak Syndrome Type 4 (HPS4) gene (OMIM: 606682) maps to human chromosome 22q12.1, and it consists of 14 exons that span approximately $32 \mathrm{~kb}$ of genomic DNA encoding an intracellular traffickingrelated cytoplasmic protein named HPS4 [11,12]. The 
human HPS4 has 708 amino acids and a predicted molecular mass of $77 \mathrm{kDa}$ and makes up BLOC-3 jointly with HPS1 as a 1:1 heterodimer [13-15]. Hermansky-Pudlak Syndrome (HPS; MIM: 203300) is a group of rare autosomal recessive diseases characterized by clinical symptoms, such as oculocutaneous albinism and bleeding diathesis, and is caused by defective biogenesis of lysosome-related organelles, such as melanosomes and platelet-dense granules [16]. In our previous case-control association study, haplotypes that were composed of five tagging single-nucleotide polymorphisms (SNPs) of HPS4 (rs4822724, rs61276843, rs9608491, rs713998, and rs2014410) were found to be significantly associated with schizophrenia, a finding that was revealed by a sliding window approach for 2-5 locus haplotypes, including rs9608491 as an essential SNP [17]. In addition, we have reported that two Japanese siblings who suffered from the comorbidity of major mental disorders, schizophrenia and major depression, and HPS harbored a nonsense mutation in HPS4 [17].

HPS is caused by a mutation in one of the genes named HPS1 to HPS9, which encode subunit proteins that consist of multiprotein complexes, the adaptor protein complex-3 (AP-3), and the biogenesis of lysosomerelated organelles complex (BLOC)-1, 2, and $3[18,19]$. BLOC-1, BLOC-2, and AP-3 interact to play a pivotal role in sorting and trafficking membrane proteins on early endosomes [20-24]. Dysbindin, which is encoded by the dystrobrevin binding protein 1 (DTNBP1) gene (also known as HPS7), is one of the subunit proteins of BLOC-1 [25]. Several lines of evidence from linkage, association, and postmortem brain studies have indicated an association between DTNBP1 and schizophrenia [26]. Genetic variants and haplotypes of DTNBP1 have frequently been reported to be associated with a number of cognitive functions, including verbal, visual, and general memory [27-30], attention [31,32], and executive function [30,32], in patients with schizophrenia and/or healthy controls. These genetic studies on DTNBP1 [26-32] led to several research studies on identification of binding partners for dysbindin [21-24]. By contrast, BLOC-3, which comprises HPS1 and HPS4, modulates the intracellular movement and distribution of late endosomes and lysosome-related organelles in cells $[14,15,33]$. Although the precise function of BLOC-3 remains to be clarified, the interaction between BLOC-3 and Rab GTPases has recently been found [34,35].

In order to investigate the association between putative candidate genes and cognitive impairments in schizophrenia, a portable instrument with a high reliability and validity is required to assess the cognition that is specifically impaired in patients with schizophrenia. Most of the neurocognitive assessment batteries that have previously been used in schizophrenia studies involve detailed assessments of the entire profile of neuropsychological function, including normal functioning, but require lengthy and complex procedures. The Brief Assessment of Cognition in Schizophrenia (BACS) was developed as a neurocognitive battery that enables brief administration (about $40 \mathrm{~min}$ ) and portability and has high reliability for specifically assessing the domains of cognitive function that are consistently impaired in schizophrenia, including verbal memory, working memory, motor speed, verbal fluency, attention, and executive function $[3,36]$. The Japanese-language version of BACS (BACS-J) has been developed for the assessment of cognition in Japanese patients with schizophrenia, and its reliability and validity have been confirmed [37].

To the best of our knowledge, HPS4 has not been reported in relation to cognitive functions in patients with schizophrenia or healthy controls. The aim of the present study was to investigate whether HPS4 is associated with cognitive functions in patients with schizophrenia and healthy controls and with the clinical profiles of patients with schizophrenia. We initially tested the association of five tagging SNPs in HPS4 with the clinical profiles and cognitive domains as measured by BACS-J in Japanese patients with schizophrenia, and the association of HPS4 SNPs with cognitive domains was also tested in healthy controls. In addition, we examined the association of HPS4 haplotypes and cognitive domains by using a sliding window approach in patients with schizophrenia and healthy controls.

\section{Methods}

\section{Subjects}

This study included 240 patients with schizophrenia (139 males $/ 101$ females; age $=48.1 \pm 12.4$, mean \pm standard deviation) and 240 unrelated healthy controls (143 males $/ 97$ females; age $=48.0 \pm 13.0$ ) who were groupmatched for age and sex. The subjects in both groups were aged 21-69 years and were recruited from the Kanto area in Japan and included a substantial portion of the subjects who were investigated in our previous genetic study [17]. The patients with schizophrenia were recruited from the Dokkyo Medical University School of Medicine Hospital and affiliated hospitals, and all of them met the Diagnostic and Statistical Manual of Mental Disorders (DSM-IV) criteria [38] for a diagnosis of schizophrenia. The onset and characteristic clinical course of schizophrenia were confirmed by examination of the medical chart. Only the patients with stable disease prior to the neurocognitive assessments were included. There were no specific medication criteria for exclusion of the patient group with respect to the administration of antipsychotics, anticholinergic drugs, and benzodiazepines. The healthy controls were unrelated volunteers who were recruited mainly from nonprofessional university/hospital 
staff, and they were screened for the absence of DSM-IV axis-I disorders. Across both groups, we excluded subjects with a history of neurological disorders, significant head injury, or significant drug or alcohol abuse. The objective of the present study was clearly explained to the participants, and written informed consent was obtained from all subjects in accordance with the Declaration of Helsinki [39]. The study was formally approved by the Institutional Review Board of the Ethical Committee of Dokkyo Medical University School of Medicine.

\section{Genotyping}

We focused on genotyping five tagging SNPs (rs4822724, rs61276843, rs9608491, rs713998, and rs2014410) in HPS4 because all the haplotypes are composed of these tagging SNPs, with rs9608491 being included as an essential SNP in the sliding window analysis of a window size of 2-5 SNPs, and these have been found to be associated with schizophrenia in our previous case-control study [17]. Genomic DNA extraction and genotyping from the peripheral blood samples that were collected from the total subjects were performed as previously described [17]. In brief, we extracted genomic DNA from the peripheral blood samples with a QIAamp DNA Blood Maxi Kit (QIAGEN Inc., Valencia, CA). Two SNPs (rs4822724 and rs9608491) were genotyped with a TaqMan ${ }^{\oplus}$ assay (Assay ID: C__2490761_10 and C_11747810_10, respectively, Applied Biosystems, Foster City, CA) with a TP800 Dice Real Time System (TaKaRa Bio Inc., Otsu, Japan). The other three SNPs (rs61276843, rs713998, and rs2014410) were genotyped with direct DNA sequencing of polymerase chain reaction products with specific primer pairs, as previously reported [17].

\section{Clinical assessment}

Smoking status and education years were self-reported by the subjects. Information on the year of disease onset was collected by chart review and double-checked by the patient. Information regarding psychopharmacological medication was collected by chart review. The dosages of individual antipsychotics were gauged based on the equivalent milligram dosage of haloperidol, and the information was summed for each patient and those of anticholinergic drugs were gauged based on the dosage of biperiden. The prescription status of anxiolytics/hypnotics was evaluated as a categorical variable. Patients were assessed according to their clinical rating of symptomatology of schizophrenia using the Positive and Negative Symptom Scale (PANSS) [40].

\section{Neurocognitive assessment}

The cognitive functions were evaluated in the subjects with version A of BACS-J [37]. The BACS-J cognitive battery consists of the following six subtests that target unique domains of cognition: list learning (verbal memory), a digit-sequencing task (working memory), a token motor task (motor speed), category fluency and letter fluency (verbal fluency), symbol coding (attention and processing speed), and the Tower of London task (TOL; executive function). The primary scores for each BACS-J subtest were transformed into z-scores whereby the healthy control mean was set to zero and its standard deviation to one. An overall composite score for global cognition was generated by averaging all of the z-scores of the six BACS-J subtests for individual participants. In addition, the subjects were assessed with the Japanese version of the National Adult Reading Test (JART) [41-43] to measure the premorbid IQs of patients with schizophrenia [44], with reference to a previous report that estimated premorbid IQ with a Wide Range Achievement Test [36].

\section{Statistical analysis}

SPSS for Windows (version 19.0; IBM Japan, Tokyo, Japan) was used for the statistical analysis. The differences in categorical variables (sex, smoking status, and prescription status of anxiolytics/hypnotics) between patients with schizophrenia and healthy controls or between the genotype groups were analyzed using a chisquare test. The demographic variables other than sex and smoking status, the clinical variables of schizophrenia other than prescription status of anxiolytics/hypnotics, and the BACS-J subtest scores were regarded as continuous variables and were assessed for normal distribution using a Shapiro-Wilk test $(P<0.05$, implying a nonnormal distribution). The differences between the groups were analyzed with analyses of variance or with the nonparametric Kruskal-Wallis and Mann-Whitney $U$-tests if the variables showed a nonnormal distribution. Departure from the Hardy-Weinberg Equilibrium was analyzed for all SNPs using the exact test that was implemented in PLINK version 1.0.7 [45,46]. SNPs with $P$ values less than 0.001 were considered to be in departure from the Hardy-Weinberg equilibrium, as described by Saito et al. [17]. The standard measures of pair-wise LD, denoted as $D^{\prime}$ and $\mathrm{r}^{2}$, were estimated in Haploview $4.2[47,48]$ based on the genotype data from all the participants.

Prior to the SNP- or haplotype-based association analyses that used general linear models, age, education years, JART-based premorbid IQ, and the BACS-J subtest scores, which showed nonnormal distributions, were standardized and normalized across all of the subjects with a mean of 0 and a variance of 1 using rank transformation according to Blom's method $[49,50]$. Associations between each HPS4 SNP and individual cognitive domains were analyzed using a linear regression model that was implemented in PLINK version 1.0.7 [45,46] with the covariates of age, sex, education years, and 
JART-based premorbid IQ because the BACS subtest performances were found to be affected by these confounding factors. The associations of each SNP with cognitive domains were tested under the three following genetic models that were fit for the minor alleles of each SNP: dominant (comparing minor allele carriers with major allele homozygotes), recessive (comparing minor allele homozygotes with major allele carriers), and additive (assuming an allelic dosage effect of minor alleles by coding none, one, or two copies of the minor allele as 0 , 1 , or 2, respectively). The associations of the haplotypes with individual cognitive domains were analyzed using haplotype-based association tests with a general linear model that was implemented in PLINK version 1.0.7 $[45,46]$ with the same covariates as were entered into the single SNP-based association analysis. A sliding window approach with a window size of 2-5 SNPs was used for the analysis. Haplotype frequencies were computed using the standard expectation-maximization algorithm, and haplotypes with a frequency of greater than $5 \%$ in the whole sample were included in the analysis. A global (or omnibus) $P$ value was calculated to jointly estimate all haplotype effects at the given locus. For both single SNP- and haplotype-based association analyses, $P$ values were calculated by running 10,000 permutations using the max $(\mathrm{T})$ procedure to correct for multiple testing between SNPs or haplotypes by each BACS-J subtest. A $P$ value less than $0.0083(0.05 / 6)$ was set as the threshold for significance in order to correct for the number of BACS-J subtests (six subtests) with Bonferroni correction. For haplotypes that met the significance criteria in the global test, a haplotype-specific test was performed to examine the independent effects of any estimated individual haplotypes by running 10,000 permutations in order to correct for multiple testing between haplotypes at the given locus.

\section{Power analysis}

A power analysis for linear regression was calculated by G*Power 3.1.6 [51] using the Linear multiple regression: Fixed model, $\mathrm{R}^{2}$ deviation from zero procedure, which provided power analyses for testing the null hypothesis that the squared multiple correlation was zero [52,53]. Our cases and controls ( $\mathrm{n}=240$, for each group) had an $80 \%$ power to detect an effect size of 0.08 or above at $\alpha=0.0083$.

\section{Results}

\section{Association of HPS4 SNPs with cognitive domains}

The detailed descriptive data for the patients with schizophrenia and the healthy controls are shown in Table 1. Both groups were well matched for age and sex. The patients with schizophrenia showed significantly lower education years $(P<0.001)$ and JART-based premorbid IQ $(P<0.001)$ and a significantly higher rate of
Table 1 Detailed descriptive data of patients with schizophrenia and healthy controls

\begin{tabular}{|c|c|c|c|c|}
\hline & $\begin{array}{c}\text { Schizophrenia } \\
(n=240) \\
\end{array}$ & $\begin{array}{l}\text { Controls } \\
(n=240) \\
\end{array}$ & $z / x^{2}$ & $P$-value \\
\hline & Mean (SD) & $\overline{\text { Mean (SD) }}$ & & \\
\hline Age (years) & $48.1(12.4)$ & $48.0(13.0)$ & -0.03 & 0.98 \\
\hline Sex $(\text { male, } \%)^{1}$ & 57.9 & 59.6 & 0.14 & 0.71 \\
\hline Education (years) & $11.7(2.1)$ & $14.2(2.3)$ & -10.85 & $<0.001$ \\
\hline Smokers $(\%)^{1}$ & 38.3 & 27.9 & 5.88 & 0.015 \\
\hline JART & $92.4(10.4)$ & $103.4(10.7)$ & -10.19 & $<0.001$ \\
\hline Age at onset (years) & $24.8(8.9)$ & - & - & - \\
\hline Antipsychotics (mg/day) ${ }^{2}$ & $12.7(9.4)$ & - & - & - \\
\hline Anticholinergics (mg/day) ${ }^{3}$ & $3.3(4.2)$ & - & - & - \\
\hline Anxiolytics/hypnotics (\%) & 75.4 & - & - & - \\
\hline \multicolumn{5}{|l|}{ PANSS } \\
\hline Positive score & $14.1(5.3)$ & - & - & - \\
\hline Negative score & $21.9(6.7)$ & - & - & - \\
\hline General score & $33.9(9.5)$ & - & - & - \\
\hline Total & $69.0(18.8)$ & - & - & - \\
\hline \multicolumn{5}{|l|}{ BACS-J scores } \\
\hline Verbal memory & $-2.01(1.31)$ & $0.00(1.00)$ & -14.63 & $<0.001$ \\
\hline Working memory & $-1.95(1.22)$ & $0.00(1.00)$ & -14.67 & $<0.001$ \\
\hline Motor speed & $-1.48(1.26)$ & $0.00(1.00)$ & -12.42 & $<0.001$ \\
\hline Verbal fluency & $-1.15(1.14)$ & $0.00(1.00)$ & -10.62 & $<0.001$ \\
\hline Attention & $-1.97(1.14)$ & $0.00(1.00)$ & -15.41 & $<0.001$ \\
\hline Executive function & $-1.77(2.26)$ & $0.00(1.00)$ & -9.67 & $<0.001$ \\
\hline Composite Score & $-1.72(1.10)$ & $0.00(0.67)$ & -15.86 & $<0.001$ \\
\hline
\end{tabular}

$P$-values were analyzed using Mann-Whitney $U$-tests, except for ${ }^{1} X^{2}$ tests. $P$-values $<0.05$ are in bold.

${ }^{2}$ Haloperidol equivalent dosage.

${ }^{3}$ Biperiden equivalent dosage.

BACS-J: Brief Assessment of Cognition in Schizophrenia,

Japanese-language version.

JART: Japanese version of the National Adult Reading Test.

PANSS: Positive and Negative Symptom Scale.

current smoking $(P=0.015)$ than controls. They were found to have significantly lower performance in all cognitive domains as measured with the $z$-scores of the six BACS-J subtests $(P<0.001)$.

The characteristics of the selected five tagging SNPs (rs4822724, rs61276843, rs9608491, rs713998, and rs2014410) are shown in Table 2. All SNPs were in Hardy-Weinberg equilibrium (all $P>0.001$ ), and they had minor allele frequencies greater than $10 \%$ in both groups. The standard LD measures, denoted as pair-wise D' and $\mathrm{r}^{2}$ measures between markers in the HPS4 gene, are illustrated in Additional file 1: Figure S1. For all SNPs, there were no significant differences in age, sex, education years, rates of current smoking, JART-based premorbid IQs, ages at onset, prescription status of anxiolytics/hypnotics, PANSS scores (positive scores, negative scores, general psychopathology scores, and total 
Table 2 Main characteristics of the selected tagging SNPs in HPS4

\begin{tabular}{|c|c|c|c|c|c|}
\hline No. & $\begin{array}{l}\text { SNP (major/ } \\
\text { minor allele) }\end{array}$ & $\begin{array}{c}\text { Localization } \\
\text { (Residue Change) }\end{array}$ & & $\begin{array}{c}\text { HWE } \\
P \text {-value }\end{array}$ & MAF \\
\hline \multirow[t]{2}{*}{1} & rs4822724 (A/G) & 5'-flanking region & Cases & 0.0139 & 0.488 \\
\hline & & & Controls & 0.2472 & 0.485 \\
\hline \multirow[t]{2}{*}{2} & rs61276843 (Del/Ins) & 5'-flanking region & Cases & 0.3959 & 0.133 \\
\hline & & & Controls & 1.0000 & 0.121 \\
\hline \multirow[t]{2}{*}{3} & rs9608491 (T/C) & intron 4 & Cases & 1.0000 & 0.179 \\
\hline & & & Controls & 0.0569 & 0.188 \\
\hline \multirow[t]{2}{*}{4} & rs713998 (G/A) & exon 9 (p.G229E) & Cases & 0.1517 & 0.233 \\
\hline & & & Controls & 0.8529 & 0.225 \\
\hline \multirow[t]{2}{*}{5} & rs2014410 (C/G) & exon 11 (p.L443V) & Cases & 0.1900 & 0.271 \\
\hline & & & Controls & 0.0014 & 0.285 \\
\hline
\end{tabular}

121-base deletion/insertion (Del/Ins) polymorphism.

HWE: Hardy-Weinberg equilibrium test.

MAF: minor allele frequency.

scores) between the genotype groups in the patients with schizophrenia (Additional file 2: Table S1 and Additional file 3: Table S2). The dosage of antipsychotics showed a significant difference between the rs9608491 genotype groups $(P=0.024)$, and the dosage of anticholinergics showed a significant difference between the rs713998 genotype groups $(P=0.031)$. The other SNP genotype groups showed no significant effects on the dosages of antipsychotics or anticholinergic drugs. In controls, there were no significant differences in any of the demographic characteristics (age, sex, education years, rates of current smoking, and JART scores) between the genotype groups of all SNPs (Additional file 4: Table S3).

The associations between each tagging SNP in HPS4 and the cognitive domains that were measured by the BACS-J subtests in the patients with schizophrenia are shown in Table 3. Under the dominant model, rs713998 showed a significant association with executive function, and this association remained after correction for multiple testing by Bonferroni correction $(\beta=0.319, \mathrm{t}=$ 3.191 , permutation $P$ value $=0.0073$ ). Under this dominant model of rs713998, there was no significant difference in dosage of anticholinergics. The patients with a minor allele carrier $(\mathrm{A} / \mathrm{A}$ and $\mathrm{A} / \mathrm{G}=-1.21 \pm 1.98$, mean \pm standard deviation) in rs713998 had a significantly higher z-score of executive function than patients with major allele homozygotes $(\mathrm{G} / \mathrm{G}=-2.14 \pm 2.36)$. In addition, rs713998 showed a nominally significant $(P<0.05)$ association with verbal memory, and this association did not remain after Bonferroni correction (verbal memory subtest score: $\mathrm{A} / \mathrm{A}$ and $\mathrm{A} / \mathrm{G}=-1.74 \pm 1.28, \mathrm{G} / \mathrm{G}=-2.19 \pm 1.31, \beta=$ $0.210, \mathrm{t}=2.704$, permutation $P$ value $=0.029$ ). There was no significant difference between the HPS4 SNPs and the cognitive domains under the recessive model or the additive model in the patients with schizophrenia.
In the controls (Table 4), rs9608491 and rs713998 were significantly associated with working memory under the recessive model, and these associations remained after correction for multiple testing with Bonferroni corrections (rs9608491, $\beta=0.701, \mathrm{t}=3.875$, permutation $P$ value $=0.001$; rs713998, $\beta=-0.646, \mathrm{t}=-3.276$, permutation $P$ value $=$ 0.0065). The healthy controls with minor homozygotes $(\mathrm{C} / \mathrm{C}=0.72 \pm 0.84)$ in rs9608491 had a higher $\mathrm{z}$-score of working memory than major allele carriers $(\mathrm{T} / \mathrm{T}$ and $\mathrm{C} / \mathrm{T}=$ $-0.04 \pm 0.99)$. Conversely, the healthy controls with minor homozygotes $(\mathrm{A} / \mathrm{A}=-1.07 \pm 0.96)$ in $\mathrm{rs} 713998$ had a lower z-score of working memory than major allele carriers (G/G and $A / G=0.05 \pm 0.97$ ). In addition, $r 4822724$ showed a nominally significant association with motor speed, and this association did not remain after Bonferroni correction (motor speed subtest score: $\mathrm{G} / \mathrm{G}=0.32 \pm 0.97, \mathrm{~A} / \mathrm{A}$ and $\mathrm{A} /$ $\mathrm{G}=-0.11 \pm 0.99, \beta=0.342, \mathrm{t}=3.027$, permutation $P$ value $=$ 0.013). There was no significant difference between HPS4 SNPs and cognitive domains under the dominant model in the healthy controls $(P>0.05)$. Under the additive model, the associations of working memory with the rs4822724, rs9608491, and rs713998 genotypes were nominally significant, and these associations did not remain after Bonferroni corrections on the additive model (z-scores of working memory subtest : $\mathrm{rs} 4822724, \mathrm{G} / \mathrm{G}=0.24 \pm 0.93, \mathrm{~A} / \mathrm{G}=0.03$ $\pm 0.97, \mathrm{~A} / \mathrm{A}=-0.27 \pm 1.06, \beta=0.148, \mathrm{t}=2.574$, permutation $P$ value $=0.047 ;$ rs9608491, $\mathrm{C} / \mathrm{C}=0.72 \pm 0.84, \mathrm{C} / \mathrm{T}=-0.06 \pm$ $1.01, \mathrm{~T} / \mathrm{T}=-0.04 \pm 0.99, \beta=0.209, \mathrm{t}=2.934$, permutation $P$ value $=0.017 ;$ rs713998, $\mathrm{A} / \mathrm{A}=-1.07 \pm 0.96, \mathrm{~A} / \mathrm{G}=-0.08$ $\pm 0.93, \mathrm{G} / \mathrm{G}=0.13 \pm 0.99, \beta=-0.217, \mathrm{t}=-3.050$, permutation $P$ value $=0.011$.

\section{Haplotype association analysis with cognitive functions}

Additional file 5: Table S4 shows the global test scores for the associations of the 2-5 locus haplotypes constructed according to the sliding window approach with individual cognitive domains in the patients with schizophrenia and the healthy controls. When the five tagging SNPs (rs4822724, rs61276843, rs9608491, rs713998, and rs2014410) were denoted with serial SNP numbers (1, 2, 3 , 4, and 5), haplotype 3-4 and 2-3-4 showed significant associations with working memory in the healthy controls, and these remained after correction for multiple testing with a Bonferroni correction (3-4: $\mathrm{t}=14.9$, permutation global $P$ value $=0.0025 ; 2-3-4: \mathrm{t}=15.0$, permutation global $P$ value $=0.0064)$. The other haplotypes, except for haplotype 1-2, showed nominal associations with working memory (permutation global $P$ value for each haplotype: $2-3,0.017 ; 4-5,0.012 ; 1-2-3,0.030 ; 3-4-5$, $0.011 ; 1-2-3-4,0.018 ; 2-3-4-5,0.013 ; 1-2-3-4-5,0.031$ ). The haplotype-specific tests of 3-4 and 2-3-4, which fulfilled the criteria for significance in the global haplotype test, with working memory were performed in the healthy controls (Table 5). In haplotype 3-4, T-A and C-G showed 
Table 3 Association of HPS4 SNPs with cognitive domains measured by the BACS-J in patients with schizophrenia

\begin{tabular}{|c|c|c|c|c|c|c|c|c|c|c|}
\hline \multirow[t]{2}{*}{ Cognitive domain } & \multirow[t]{2}{*}{ SNP } & \multicolumn{3}{|c|}{ Dominant model } & \multicolumn{3}{|c|}{ Recessive model } & \multicolumn{3}{|c|}{ Additive model } \\
\hline & & $\beta$ & $t$ & $P$ (perm) & $\beta$ & $t$ & $P$ (perm) & $\beta$ & $t$ & $P($ perm $)$ \\
\hline \multirow[t]{5}{*}{ Verbal memory } & rs4822724 & -0.028 & -0.296 & 0.999 & -0.031 & -0.323 & 0.998 & -0.023 & -0.388 & 0.991 \\
\hline & rs61276843 & 0.183 & 2.062 & 0.165 & 0.275 & 1.120 & 0.757 & 0.164 & 2.139 & 0.131 \\
\hline & rs9608491 & -0.040 & -0.489 & 0.989 & 0.147 & 0.644 & 0.967 & -0.016 & -0.226 & 0.999 \\
\hline & rs713998 & 0.210 & 2.704 & 0.029 & 0.097 & 0.644 & 0.967 & 0.146 & 2.386 & 0.074 \\
\hline & rs2014410 & -0.128 & -1.651 & 0.360 & -0.236 & -1.388 & 0.569 & -0.123 & -1.912 & 0.212 \\
\hline \multirow[t]{5}{*}{ Working memory } & rs4822724 & 0.183 & 1.882 & 0.243 & -0.026 & -0.255 & 1.000 & 0.065 & 1.037 & 0.746 \\
\hline & rs61276843 & 0.024 & 0.259 & 1.000 & -0.123 & -0.474 & 0.992 & 0.006 & 0.076 & 1.000 \\
\hline & rs9608491 & 0.117 & 1.370 & 0.554 & 0.254 & 1.063 & 0.797 & 0.115 & 1.535 & 0.406 \\
\hline & rs713998 & 0.068 & 0.824 & 0.896 & -0.091 & -0.580 & 0.982 & 0.026 & 0.408 & 0.988 \\
\hline & rs2014410 & -0.012 & -0.141 & 1.000 & -0.313 & -1.753 & 0.329 & -0.053 & -0.781 & 0.888 \\
\hline \multirow[t]{5}{*}{ Motor speed } & rs4822724 & 0.043 & 0.356 & 0.997 & 0.110 & 0.883 & 0.887 & 0.059 & 0.770 & 0.895 \\
\hline & rs61276843 & -0.079 & -0.685 & 0.951 & 0.038 & 0.118 & 1.000 & -0.056 & -0.555 & 0.969 \\
\hline & rs9608491 & 0.193 & 1.830 & 0.260 & 0.290 & 0.983 & 0.838 & 0.177 & 1.912 & 0.215 \\
\hline & rs713998 & -0.081 & -0.797 & 0.910 & 0.093 & 0.476 & 0.992 & -0.035 & -0.430 & 0.988 \\
\hline & rs2014410 & 0.069 & 0.683 & 0.952 & -0.169 & -0.763 & 0.936 & 0.024 & 0.280 & 0.998 \\
\hline \multirow[t]{5}{*}{ Verbal fluency } & rs4822724 & -0.087 & -0.680 & 0.947 & -0.036 & -0.272 & 1.000 & -0.049 & -0.601 & 0.956 \\
\hline & rs61276843 & 0.061 & 0.500 & 0.987 & -0.066 & -0.196 & 1.000 & 0.039 & 0.371 & 0.993 \\
\hline & rs9608491 & 0.047 & 0.421 & 0.993 & 0.026 & 0.083 & 1.000 & 0.039 & 0.394 & 0.991 \\
\hline & rs713998 & 0.152 & 1.412 & 0.524 & 0.210 & 1.016 & 0.820 & 0.129 & 1.530 & 0.419 \\
\hline & rs2014410 & -0.050 & -0.473 & 0.989 & -0.102 & -0.432 & 0.995 & -0.050 & -0.559 & 0.966 \\
\hline \multirow[t]{5}{*}{ Attention } & rs4822724 & -0.075 & -0.917 & 0.855 & 0.016 & 0.181 & 1.000 & -0.025 & -0.473 & 0.982 \\
\hline & rs61276843 & 0.065 & 0.815 & 0.903 & 0.099 & 0.453 & 0.994 & 0.058 & 0.847 & 0.858 \\
\hline & rs9608491 & 0.045 & 0.625 & 0.965 & 0.094 & 0.464 & 0.993 & 0.044 & 0.692 & 0.924 \\
\hline & rs713998 & 0.123 & 1.778 & 0.294 & 0.125 & 0.937 & 0.870 & 0.097 & 1.785 & 0.265 \\
\hline & rs2014410 & -0.051 & -0.738 & 0.932 & -0.132 & -0.871 & 0.897 & -0.055 & -0.948 & 0.803 \\
\hline \multirow[t]{5}{*}{ Executive function } & rs4822724 & -0.132 & -1.096 & 0.740 & -0.132 & -1.057 & 0.798 & -0.104 & -1.352 & 0.522 \\
\hline & rs61276843 & 0.257 & 2.242 & 0.111 & -0.373 & -1.169 & 0.730 & 0.156 & 1.561 & 0.390 \\
\hline & rs9608491 & -0.054 & -0.511 & 0.985 & -0.150 & -0.506 & 0.991 & -0.056 & -0.606 & 0.953 \\
\hline & rs713998 & 0.319 & 3.191 & $0.0073^{*}$ & -0.050 & -0.259 & 1.000 & 0.188 & 2.377 & 0.080 \\
\hline & rs2014410 & -0.101 & -1.002 & 0.797 & -0.251 & -1.133 & 0.752 & -0.107 & -1.269 & 0.578 \\
\hline
\end{tabular}

Linear regression analyses were performed with the covariates of age, sex, education years, and JART scores. Permutation $P$-values, $P$ (perm), were adjusted for multiple testing between SNPs by each BACS-J subtest $P$-values $<0.0083(0.05 / 6)$ were considered as statistically significant to correct for the number of BACS- $J$ subtests. $P$-values $<0.05$ are in bold, and statistically significant results are marked with **'.

BACS-J: Brief Assessment of Cognition in Schizophrenia, Japanese-language version.

significant negative and positive effects, respectively, on working memory (T-A: $\beta=-0.216, t=9.30$, permutation specific $P$ value $=0.0074 ; \mathrm{C}-\mathrm{G}: \beta=0.209, \mathrm{t}=8.61$, permutation specific $P$ value $=0.011$ ). In haplotype $2-3-4$, Del-C$G$ showed a significant positive effect on working memory (Del-C-G: $\beta=0.209, \mathrm{t}=8.61$, permutation specific $P$ value $=0.014)$. Del-C-G and C-G occurred at comparable frequencies, as did Del-T-G and T-G, indicating that haplotype 2-3-4 containing the major allele $(G)$ at rs713998 was tagged most effectively by the major allele (del) at rs61276843. These associations remained significant after Bonferroni correction $[P<0.025(0.05 / 2)]$ for multiple testing of the two classes (3-4 and 2-3-4) of the tested haplotypes.

\section{Discussion}

In the present study, which was based on our previous reports indicating an association of HPS4 with schizophrenia [17], we evaluated the associations of HPS4 with the clinical profiles of patients with schizophrenia and the cognitive functions of patients with schizophrenia and healthy controls. The major findings of our study were as follows: (1) the HPS4 rs713998 polymorphism was significantly associated with executive function in 
Table 4 Association of HPS4 SNPs with cognitive domains measured by the BACS-J in healthy controls

\begin{tabular}{|c|c|c|c|c|c|c|c|c|c|c|}
\hline \multirow[t]{2}{*}{ Cognitive domain } & \multirow[t]{2}{*}{ SNP } & \multicolumn{3}{|c|}{ Dominant model } & \multicolumn{3}{|c|}{ Recessive model } & \multicolumn{3}{|c|}{ Additive model } \\
\hline & & $\beta$ & $t$ & $P$ (perm) & $\beta$ & $t$ & $P($ perm $)$ & $\beta$ & $t$ & $P($ perm $)$ \\
\hline \multirow[t]{5}{*}{ Verbal memory } & rs4822724 & 0.075 & 0.870 & 0.873 & 0.147 & 1.670 & 0.380 & 0.081 & 1.539 & 0.402 \\
\hline & rs61276843 & -0.071 & -0.777 & 0.913 & -0.072 & -0.208 & 1.000 & -0.065 & -0.767 & 0.890 \\
\hline & rs9608491 & 0.029 & 0.352 & 0.997 & 0.303 & 1.798 & 0.304 & 0.064 & 0.975 & 0.778 \\
\hline & rs713998 & -0.134 & -1.724 & 0.317 & -0.124 & -0.675 & 0.967 & -0.111 & -1.700 & 0.310 \\
\hline & rs2014410 & 0.014 & 0.182 & 1.000 & 0.010 & 0.085 & 1.000 & 0.009 & 0.169 & 1.000 \\
\hline \multirow[t]{5}{*}{ Working memory } & rs4822724 & 0.225 & 2.412 & 0.069 & 0.174 & 1.793 & 0.313 & 0.148 & 2.574 & 0.047 \\
\hline & rs61276843 & -0.150 & -1.494 & 0.459 & -0.882 & -2.361 & 0.086 & -0.181 & -1.961 & 0.193 \\
\hline & rs9608491 & 0.162 & 1.797 & 0.283 & 0.701 & 3.875 & $0.001 *$ & 0.209 & 2.934 & 0.017 \\
\hline & rs713998 & -0.187 & -2.202 & 0.120 & -0.646 & -3.276 & $0.0065^{*}$ & -0.217 & -3.050 & 0.011 \\
\hline & rs2014410 & -0.069 & -0.824 & 0.893 & 0.096 & 0.747 & 0.945 & -0.014 & -0.236 & 0.999 \\
\hline \multirow[t]{5}{*}{ Motor speed } & rs4822724 & 0.085 & 0.764 & 0.921 & 0.342 & 3.027 & 0.013 & 0.155 & 2.274 & 0.095 \\
\hline & rs61276843 & -0.179 & -1.502 & 0.458 & -0.280 & -0.626 & 0.973 & -0.169 & -1.539 & 0.399 \\
\hline & rs9608491 & 0.045 & 0.417 & 0.994 & 0.159 & 0.722 & 0.951 & 0.052 & 0.613 & 0.951 \\
\hline & rs713998 & -0.127 & -1.259 & 0.627 & -0.271 & -1.139 & 0.749 & -0.126 & -1.475 & 0.439 \\
\hline & rs2014410 & -0.090 & -0.897 & 0.863 & -0.075 & -0.495 & 0.992 & -0.062 & -0.869 & 0.844 \\
\hline \multirow[t]{5}{*}{ Verbal fluency } & rs4822724 & -0.048 & -0.487 & 0.989 & 0.051 & 0.500 & 0.991 & $-7.1 \times 10^{-5}$ & -0.001 & 1.000 \\
\hline & rs61276843 & -0.217 & -2.084 & 0.168 & -0.574 & -1.469 & 0.522 & -0.219 & -2.287 & 0.092 \\
\hline & rs9608491 & -0.149 & -1.594 & 0.402 & 0.062 & 0.321 & 1.000 & -0.086 & -1.145 & 0.664 \\
\hline & rs713998 & -0.171 & -1.935 & 0.221 & -0.160 & -0.763 & 0.942 & -0.143 & -1.911 & 0.213 \\
\hline & rs2014410 & 0.174 & 1.993 & 0.199 & 0.051 & 0.379 & 0.998 & 0.099 & 1.590 & 0.373 \\
\hline \multirow[t]{5}{*}{ Attention } & rs4822724 & 0.090 & 1.171 & 0.697 & 0.063 & 0.793 & 0.929 & 0.057 & 1.199 & 0.629 \\
\hline & rs61276843 & -0.141 & -1.718 & 0.326 & -0.359 & -1.168 & 0.728 & -0.141 & -1.873 & 0.226 \\
\hline & rs9608491 & 0.062 & 0.842 & 0.892 & 0.229 & 1.508 & 0.481 & 0.074 & 1.257 & 0.585 \\
\hline & rs713998 & -0.044 & -0.629 & 0.962 & -0.226 & -1.378 & 0.575 & -0.060 & -1.026 & 0.744 \\
\hline & rs2014410 & -0.008 & -0.116 & 1.000 & -0.035 & -0.335 & 0.998 & -0.004 & -0.074 & 1.000 \\
\hline \multirow[t]{5}{*}{ Executive function } & rs4822724 & 0.003 & 0.032 & 1.000 & 0.039 & 0.381 & 0.997 & 0.015 & 0.248 & 0.998 \\
\hline & rs61276843 & -0.049 & -0.468 & 0.987 & 0.183 & 0.464 & 0.993 & -0.031 & -0.316 & 0.996 \\
\hline & rs9608491 & 0.003 & 0.035 & 1.000 & -0.066 & -0.339 & 0.998 & -0.008 & -0.104 & 1.000 \\
\hline & rs713998 & 0.020 & 0.229 & 1.000 & 0.099 & 0.470 & 0.993 & 0.027 & 0.363 & 0.993 \\
\hline & rs2014410 & -0.049 & -0.558 & 0.976 & -0.057 & -0.423 & 0.996 & -0.037 & -0.594 & 0.954 \\
\hline
\end{tabular}

Linear regression analyses were performed with the covariates of age, sex, education years, and JART scores. Permutation $P$-values, $P$ (perm), were adjusted for multiple testing between SNPs by each BACS-J subtest $P$-values $<0.0083(0.05 / 6)$ were considered as statistically significant to correct for the number of BACS- $J$ subtests. $P$-values $<0.05$ are in bold, and statistically significant results are marked with “*”.

BACS-J: Brief Assessment of Cognition in Schizophrenia, Japanese-language version.

Japanese patients with schizophrenia, and (2) the polymorphisms (rs9608491 and rs713998) and haplotypic variants (rs9608491-713998 and rs61276843-9608491713998) in HPS4 were significantly associated with working memory in Japanese healthy controls. These significances survived after corrections for multiple testing. Associations of the clinical profiles of patients with schizophrenia (PANSS scores, ages at onset, and premorbid IQs as measured by JART) with each SNP in HPS4 were not observed.

Culminating lines of evidence have suggested that patients with schizophrenia show deficits of executive function, which is mainly mediated by the frontal lobe and which is involved in the ability of goal formation, planning, executing goal-directed plans, and effective performance $[4,54]$. The TOL task, which was employed as a task in order to assess executive function in the BACS [3], is known to be involved in multifaceted aspects of executive function, including working memory, planning, problem-solving, and inhibition $[55,56]$. Task complexity in performing the TOL correlated with activation of the dorsolateral prefrontal cortex, the lateral premotor cortex, the rostral anterior cingulate cortex, and the right dorsal caudate nucleus in a positron 
Table 5 Association of working memory with specific haplotypes of HPS4 in healthy controls

\begin{tabular}{lccccc}
\hline Haplotype $^{1}$ & & Frequency & $\boldsymbol{\beta}$ & $\mathbf{t}$ & Specific $\boldsymbol{P}$ (perm) \\
\hline $3-4$ & T-G & 0.588 & 0.005 & 0.008 & 0.996 \\
& T-A & 0.225 & -0.216 & 9.30 & $\mathbf{0 . 0 0 7 4}$ \\
2-3-4 & C-G & 0.187 & 0.209 & 8.61 & $\mathbf{0 . 0 1 1 ^ { * }}$ \\
& Del-T-G & 0.585 & 0.006 & 0.012 & 0.999 \\
& Del-C-G & 0.187 & 0.209 & 8.61 & $\mathbf{0 . 0 1 4 ^ { * }}$ \\
& Del-T-A & 0.107 & -0.194 & 4.11 & 0.141 \\
& Ins-T-A & 0.118 & -0.180 & 3.75 & 0.171 \\
\hline
\end{tabular}

Haplotype-specific tests with general linear models were performed with the covariates of age, sex, education years, and JART scores. Permutation specific $P$-values, specific $P$ (perm), were adjusted for multiple testing between individual haplotypes at the given locus $P$-values $<0.025(0.05 / 2)$ were considered as statistically significant to correct for the number of tested haplotypes (3-4 and 2-3-4). $P$-values $<0.05$ are in bold, and statistically significant results are marked with '*'.

${ }^{1}$ SNP numbers are as follows: 2 = rs61276843, 3 = rs9608491, 4 = rs713998.

emission tomographic study of healthy subjects [57]. Moreover, decreased prefrontal activation during the TOL has been reported in subjects with first-episode schizophrenia [58]. We showed that the minor allele A that is carried at rs713998 was significantly associated with higher performance in executive function that was measured by the TOL task in patients with schizophrenia under the dominant genetic model, but there was no association between any of the HPS4 SNPs and executive function in healthy controls. Because the sample size, age, and percentage of males were almost comparable between the patients with schizophrenia and the healthy controls, these results suggested that the rs713998 polymorphism confers distinct properties in frontal cortexmediated executive function, specifically in patients with schizophrenia.

HS4 is unique in binding to the counterpart protein HPS1 and in forming a 1:1 heterodimer called BLOC-3 that is involved in late endosomal trafficking [14,15,33]. Both the N-terminal (amino acid: 1-274) and C-terminal domains (534-708) of HPS4 have been found to be required for assembly with a full-length HPS1 to form a soluble and stable BLOC-3 complex [34]. Rs713998 is a nonsynonymous SNP that is located in exon 9 (p.G229E) and that has been reported to be completely linked with three other nonsynonymous SNPs (rs5752330: exon 11, p. M552V; rs1894706: exon 12, p.Y606H; rs1894704: exon 13, p.H625G) of HPS4 [17]. Given that the four SNPs (rs713998, rs5752330, rs1894706, and rs1894704) that completely link with each other occur in domains that are required for HPS1-HPS4 interaction (N-terminal domain: rs713998; C-terminal domain: rs5752330, rs1894706, and rs1894704), it is plausible that the amino-acid substitutions that are caused by these SNPs affect the HPS1HPS4 interactions and BLOC-3 complex stability. The HPS1-HPS4 interaction is also indispensable for the recently identified function of BLOC-3 as a guanine nucleotide exchange factor (GEF) for the small GTPases Rab32/38 [35]. Although the longin domain that was bioinformatically predicted to occur in the $\mathrm{N}$-terminal portion of HPS4 may be implicated in Rab GEF activity [35], no exonic tagging SNP corresponding to this domain was included in the present study.

Working memory impairments have been consistently reported in patients with schizophrenia, even those with first-episode schizophrenia with highly preserved IQ [59]. A digit-sequencing task, which has been employed as a task for assessing working memory in BACS [3], is akin to a number-letter sequencing task. The latter was found to activate the dorsolateral prefrontal cortex, the orbital frontal lobe, the premotor cortex, and the posterior parietal cortex, mainly in the right hemisphere, in a positron emission tomographic study of healthy subjects [60]. An SNP-based association analysis that was conducted under the recessive model and a haplotype-based association analysis have consistently shown that the Callele at rs9608491 and the G-allele at 713998 conferred high performance in working memory in healthy controls. Rs9608491 would contribute to a greater proportion of the effect of this two-marker haplotype in the performance of working memory in this population due to its higher regression coefficient and smaller $P$ value $(\beta=0.701, P$ value $=0.001)$ than rs713998 under the recessive model. However, the finding of the involvement of rs713998 in working memory is apparently paradoxical because rs713998 at the A-allele state exerts a beneficial effect on executive function in patients with schizophrenia. Thus, working memory and executive function that is measured by the TOL may reflect different aspects of cognition, even though they are intimately related to each other, and rs713998 may exert its effects on these cognitive subdomains across controls and patients. Moreover, the finding that two alleles carried at rs713998 showed differential effects on cognitive aspects in cases versus controls could indicate a kind of ceiling effect that cannot be accounted for by a simple dose-response relationship of the alleles. For example, it has been suggested that the catechol-O-methyltransferase Val158Met polymorphism has an opposite effect on verbal fluency in healthy controls compared with patients with schizophrenia [61]. In patients with schizophrenia, there has been no association of working memory with any polymorphism or haplotype that has been examined in HPS4, although medication and clinical symptoms may have the potential to obscure any effects of genetic variance. We previously found that protective haplotypes always contained the C-allele carried at rs9608491 in HPS4 in a case-control study [17]. As discussed in our previous study [17], an argument for the involvement of variants of HPS4, particularly rs9608491, in the susceptibility to 
schizophrenia awaits further research. Collectively, the $\mathrm{C}$-allele at rs9608491 may have a certain role in protecting against the susceptibility to schizophrenia and in enhancing working memory performance in controls only.

Very little information has thus far been found regarding the neurobiological and behavioral aspects of HPS4, although it is ubiquitously expressed in most tissues, including brain [11]. To the best of our knowledge, the Allen Brain Atlas [62] has only shown that HPS4 is expressed in the hippocampal formation, the olfactory areas, the cortical subplate, and the cerebellum of the male adult mouse using in situ hybridization. Notwithstanding the uncertainty about the role of this gene in these brain regions, the data obtained here regarding the association between rs9608491 and working memory suggested that HPS4 plays a certain role in working memory that is driven by the appropriate function of the prefrontal cortex. Further analysis is required to reveal a putative role of HPS4 in the neurobiology related to the cognitive deficits in schizophrenia.

Several limitations of the present study must be mentioned. First, although the total sample size was moderate for an association study of cognitive functions, a relatively small number of individuals were included as carriers of minor homozygotes for each SNP, except for rs4822724. This could have resulted in a selection bias and decreased the power under the recessive model when individuals carrying the minor homozygotes were assigned to a distinct group. The associations of working memory with rs9608491 and rs713998 in the healthy controls was revealed by the recessive model, which yielded a minor homozygote group with a small number of individuals (rs9608491 C/C, n=13; rs713998 A/A, n=11). Replication of these findings in an independent large cohort or in different ethnic cohorts is needed. Second, several lines of evidence have indicated that cognitive performance in patients with schizophrenia is influenced by psychopharmacological medications $[63,64]$. In our positive finding of an association between rs713998 and executive function in patients with schizophrenia, we confirmed there was no significant difference $(P>0.05)$ in the dosages of antipsychotics, anticholinergics, or the prescription status of anxiolytics/hypnotics under the dominant model. However, in a real-world clinical setting, the extent to which various kinds of antipsychotics or their combination, particularly in the context of the polypharmacy of antipsychotic medication, exerts an effect, if any, on the cognitive performance of patents with schizophrenia is difficult to analyze. Third, rs9608491 is an intronic SNP located in between the exons encoding the $\mathrm{N}$-terminal domain. Its function is uncertain because it is unlikely to affect the stability of HPS4 mRNA [17]. This is reminiscent of the relevant SNPs of the DTNBP1 gene, which are mostly intronic and have been significantly implicated in the executive function of healthy controls [30], in cases belonging to early-onset families with functional psychosis disorders [65], and in the spatial working memory in patients with schizophrenia [66]. This general aspect regarding the question as to why intronic SNPs are involved in cognitive function awaits further genetic study.

\section{Conclusions}

Our present study demonstrated that genetic variants in HPS4 were associated with executive function in patients with schizophrenia and with working memory in healthy controls. To the best of our knowledge, this is the first report of an association of HPS4 with cognitive functions and the first to employ the BACS-J as a battery in a SNP- and haplotype-based association study of cognitive functions. Our present results suggested that HPS4 may be involved in the executive function deficits of patients with schizophrenia. Further research is needed to elucidate the role of HPS4 in higher brain functions through neurobiological and behavioral analyses of animal models.

\section{Additional files}

Additional file 1: Figure S1. Linkage disequilibrium (LD) block structure of the HPS4 gene. The values in the boxes represent the pair-wise D'and $r^{2}$ measures between markers. Boxes without values indicate $\mid D^{\prime}$-value $\mid=1.00$.

Additional file 2: Table S1. Demographics for each genotype group of HPS4 SNPs in patients with schizophrenia.

Additional file 3: Table S2. Clinical characteristics for each genotype group of HPS4 SNPs in patients with schizophrenia.

Additional file 4: Table S3. Demographics for each genotype group of HPS4 SNPS in healthy controls.

Additional file 5: Table S4. Global haplotype association for 2-5 SNPS in HPS4 with cognitive domains in patients and controls.

\section{Competing interests}

Kazufumi Akiyama is a paid consultant to Taisho Toyama Pharmaceutical Co., Ltd. This consultancy had no further role in the study design, the collection, analysis and interpretation of data, the writing of the report, or the decision to submit the paper for publication. None of the remaining authors declare any conflicts of interest.

\section{Authors' contributions}

GK was involved in the design of the study, performed the statistical analysis, and drafted the manuscript. AS was involved in the design of the study, sample collection, and genotyping. YO, TW, KF, KS, TI, HM and KO were involved in participation in the design of the study and coordination for sample collection. KA was involved in the design of the study, laboratory coordination, sample collection, and editing the manuscript. All authors read and approved final manuscript.

\section{Acknowledgements}

This work was supported by the Japan Society for the Promotion of Science (JSPS) KAKENHI Grant number: 23791351 to Go Kuratomi in the fiscal year

2011-2013. This funding source had no further role in the study design, the collection, analysis, and interpretation of the data, the writing of the report; or the decision to submit the paper for publication. No other funding supported any aspect of the submitted work. The authors thank Ms. Mikiko Ishikawa and Sachiko Suzuki for their excellent technical assistance. 


\section{Author details}

${ }^{1}$ Department of Biological Psychiatry and Neuroscience, Dokkyo Medical University School of Medicine, 880 Kitakobayashi, Mibu, Tochigi 3210293, Japan. 2Department of Psychiatry, Dokkyo Medical University School of Medicine, 880 Kitakobayashi, Mibu, Tochigi 3210293, Japan. ${ }^{3}$ Department of Internal Medicine (Endocrinology, Metabolism, and Hematology), Dokkyo Medical University Koshigaya Hospital, 2-1-50 Minamikoshigaya, Koshigaya, Saitama 3438555, Japan. ${ }^{4}$ Mori Hospital, 419 lidamachi, Utsunomiya, Tochigi 3210347, Japan. ${ }^{5}$ Takizawa Hospital, 2-29 Hanabusahoncho Utsunomiya, Tochigi 3200828, Japan.

Received: 27 August 2013 Accepted: 25 October 2013

Published: 30 October 2013

\section{References}

1. Green MF, Kern RS, Braff DL, Mintz J: Neurocognitive deficits and functional outcome in schizophrenia: are we measuring the "right stuff"? Schizophr Bull 2000, 26(1):119-136.

2. Gold JM: Cognitive deficits as treatment targets in schizophrenia. Schizophr Res 2004, 72(1):21-28.

3. Keefe RS, Goldberg TE, Harvey PD, Gold JM, Poe MP, Coughenour L: The Brief Assessment of Cognition in Schizophrenia: reliability, sensitivity, and comparison with a standard neurocognitive battery. Schizophr Res 2004, 68(2-3):283-297.

4. Eisenberg DP, Berman KF: Executive function, neural circuitry, and genetic mechanisms in schizophrenia. Neuropsychopharmacology 2010, 35(1):258-277.

5. Gur RE, Calkins ME, Gur RC, Horan WP, Nuechterlein KH, Seidman L, Stone WS: The Consortium on the Genetics of Schizophrenia: neurocognitive endophenotypes. Schizophr Bull 2007, 33(1):49-68.

6. Green AE, Munafò MR, DeYoung CG, Fossella JA, Fan J, Gray JR: Using genetic data in cognitive neuroscience: from growing pains to genuine insights. Nat Rev Neurosci 2008, 9(9):710-720.

7. Meyer-Lindenberg A, Weinberger DR: Intermediate phenotypes and genetic mechanisms of psychiatric disorders. Nat Rev Neurosci 2006, 7(10):818-827.

8. DeLisi LE, Shaw SH, Crow TJ, Shields G, Smith AB, Larach W, Wellman N Loftus J, Nanthakumar B, Razi K, Stewart J, Comazzi M, Vita A, Heffner T, Sherrington R: A genome-wide scan for linkage to chromosomal regions in 382 sibling pairs with schizophrenia or schizoaffective disorder. Am J Psychiatry 2002, 159(5):803-812.

9. Gill M, Vallada $H$, Collier D, Sham P, Holmans P, Murray R, McGuffin P, Nanko S, Owen M, Antonarakis S, Housman D, Kazazian H, Nestadt G, Pulver AE, Straub RE, MacLean CJ, Walsh D, Kendler KS, DeLisi L, Polymeropoulos M, Coon H, Byerley W, Lofthouse R, Gershon E, Golden L, Crow T, Freedman R, Laurent C, Bodeau-Pean S, d'Amato T, et al: A combined analysis of D22S278 marker alleles in affected sib-pairs: Support for a susceptibility locus for schizophrenia at chromosome 22q12. Am J Med Genet 1996, 67(1):40-45.

10. Takahashi S, Ohtsuki T, Yu SY, Tanabe E, Yara K, Kamioka M, Matsushima E, Matsuura M, Ishikawa K, Minowa Y, Noguchi E, Nakayama J, YamakawaKobayashi K, Arinami T, Kojima T: Significant linkage to chromosome 22q for exploratory eye movement dysfunction in schizophrenia. Am J Med Genet Part B 2003, 123B(1):27-32.

11. Anderson PD, Huizing M, Claassen DA, White J, Gahl WA: HermanskyPudlak syndrome type 4 (HPS-4): clinical and molecular characteristics. Hum Genet 2003, 113(1):10-17.

12. Suzuki T, Li W, Zhang Q, Karim A, Novak EK, Sviderskaya EV, Hill SP, Bennett DC, Levin AV, Nieuwenhuis HK, Fong CT, Castellan C, Miterski B, Swank RT, Spritz RA Hermansky-Pudlak syndrome is caused by mutations in HPS4, the human homolog of the mouse light-ear gene. Nat Genet 2002, 30(3):321-324.

13. Chiang PW, Oiso N, Gautam R, Suzuki T, Swank RT, Spritz RA: The HermanskyPudlak syndrome 1 (HPS1) and HPS4 proteins are components of two complexes, BLOC-3 and BLOC-4, involved in the biogenesis of lysosomerelated organelles. J Biol Chem 2003, 278(22):20332-20337.

14. Martina JA, Moriyama K, Bonifacino JS: BLOC-3, a protein complex containing the Hermansky-Pudlak syndrome gene products HPS1 and HPS4. J Biol Chem 2003, 278(31):29376-29384.

15. Nazarian R, Falcón-Pérez JM, Dell'Angelica EC: Biogenesis of lysosome-related organelles complex 3 (BLOC-3): a complex containing the HermanskyPudlak syndrome (HPS) proteins HPS1 and HPS4. Proc Natl Acad Sci USA 2003, 100(15):8770-8775.

16. Hermansky F, Pudlak P: Albinism associated with hemorrhagic diathesis and unusual pigmented reticular cells in the bone marrow: report of two cases with histochemical studies. Blood 1959, 14(2):162-169.
17. Saito A, Kuratomi G, Ito C, Matsuoka H, Suzuki T, Ozeki Y, Watanabe T, Fujii K, Shimoda K, Fukushima Y, Inukai T, Ohmori K, Akiyama K: An association study of the Hermansky-Pudlak syndrome type 4 gene in schizophrenic patients. Psychiatr Genet 2013, 23(4):163-173.

18. Cullinane AR, Curry JA, Carmona-Rivera C, Summers CG, Ciccone C, Cardillo ND, Dorward H, Hess RA, White JG, Adams D, Huizing M, Gahl WA: A BLOC1 mutation screen reveals that PLDN is mutated in Hermansky-Pudlak Syndrome type 9. Am J Hum Genet 2011, 88(6):778-787.

19. Wei ML: Hermansky-Pudlak syndrome: a disease of protein trafficking and organelle function. Pigment Cell Res 2006, 19(1):19-42.

20. Di Pietro SM, Falcón-Pérez JM, Tenza D, Setty SR, Marks MS, Raposo G, Dell'Angelica EC: BLOC-1 interacts with BLOC-2 and the AP-3 complex to facilitate protein trafficking on endosomes. Mol Biol Cell 2006, 17(9):4027-4038.

21. Hikita T, Taya S, Fujino Y, Taneichi-Kuroda S, Ohta K, Tsuboi D, Shinoda T, Kuroda K, Funahashi Y, Uraguchi-Asaki J, Hashimoto R, Kaibuchi K: Proteomic analysis reveals novel binding partners of dysbindin, a schizophrenia-related protein. J Neurochem 2009, 110(5):1567-1574

22. Taneichi-Kuroda S, Taya S, Hikita T, Fujino Y, Kaibuchi K: Direct interaction of dysbindin with the AP-3 complex via its mu subunit. Neurochem Int 2009, 54(7):431-438

23. Larimore J, Tornieri K, Ryder PV, Gokhale A, Ziatic SA, Craige B, Lee JD, Talbot K, Pare J-F, Smith Y, Faundez V: The schizophrenia susceptibility factor dysbindin and its assciated complex sort cargoes from cell bodies to the synapse. Mol Biol Cell 2011, 22(24):4854-4867.

24. Gokhale A, Larimore J, Werner E, So L, Moreno-De-Luca A, Lese-Martin C, Lupashin W, Smith Y, Faundez V: Quantitative proteomic and genetic analyses of the schizophrenia susceptibility factoe dysbindin identify novel roles of the biogenesis of lysosome-related organelles complex 1. J Neurosci 2012, 32(11):3697-3711.

25. Li W, Zhang Q, Oiso N, Novak EK, Gautam R, O'Brien EP, Tinsley CL, Blake DJ, Spritz RA, Copeland NG, Jenkins NA, Amato D, Roe BA, Starcevic M, Dell'Angelica EC, Elliott RW, Mishra V, Kingsmore SF, Paylor RE, Swank RT: Hermansky-Pudlak syndrome type 7 (HPS-7) results from mutant dysbindin, a member of the biogenesis of lysosome-related organelles complex 1 (BLOC-1). Nat Genet 2003, 35(1):84-89.

26. Mullin AP, Gokhale A, Larimore J, Faundez V: Cell biology of the BLOC-1 complex subunit dysbindin, a schizophrenia susceptibility gene. Mol Neurobiol 2011, 44(1):53-64.

27. Alfimova MV, Monakhov MV, Abramova LI, Golubev SA, Golimbet VE: Polymorphism of serotonin receptor genes (5-HTR2A) and Dysbindin (DTNBP1) and individual components of short-term verbal memory processes in Schizophrenia. Neurosci Behav Physiol 2010, 40(8):934-940.

28. Hashimoto R, Noguchi H, Hori H, Ohi K, Yasuda Y, Takeda M, Kunugi H: Association between the dysbindin gene (DTNBP1) and cognitive functions in Japanese subjects. Psychiatry Clin Neurosci 2009, 63(4):550-556.

29. Hashimoto R, Noguchi H, Hori H, Nakabayashi T, Suzuki T, Iwata N, Ozaki N, Kosuga A, Tatsumi M, Kamijima K, Harada S, Takeda M, Saitoh O, Kunugi H: A genetic variation in the dysbindin gene (DTNBP1) is associated with memory performance in healthy controls. World J Biol Psychiatry 2010, 11(2):431-438.

30. Luciano M, Miyajima F, Lind PA, Bates TC, Horan M, Harris SE, Wright MJ, Ollier WE, Hayward C, Pendleton N, Gow AJ, Visscher PM, Starr JM, Deary IJ, Martin NG, Payton A: Variation in the dysbindin gene and normal cognitive function in three independent population samples. Genes Brain Behav 2009, 8(2):218-227.

31. Baek JH, Kim JS, Ryu S, Oh S, Noh J, Lee WK, Park T, Lee YS, Lee D, Kwon JS, Hong KS: Association of genetic variations in DTNBP1 with cognitive function in schizophrenia patients and healthy subjects. Am J Med Genet Part B 2012, 159B(7):841-849

32. Stefanis NC, Trikalinos TA, Avramopoulos D, Smyrnis N, Evdokimidis I, Ntzani EE, loannidis JP, Stefanis CN: Impact of schizophrenia candidate genes on schizotypy and cognitive endophenotypes at the population level. Biol Psychiatry 2007, 62(7):784-792.

33. Falcón-Pérez JM, Nazarian R, Sabatti C, Dell'Angelica EC: Distribution and dynamics of Lamp1-containing endocytic organelles in fibroblasts deficient in BLOC-3. J Cell Sci 2005, 118(22):5243-5255.

34. Kloer DP, Rojas R, Ivan V, Moriyama K, van Vlijmen T, Murthy N, Ghirlando R, van der Sluijs P, Hurley JH, Bonifacino JS: Assembly of the biogenesis of lysosome-related organelles complex-3 (BLOC-3) and its interaction with Rab9. J Biol Chem 2010, 285(10):7794-7804.

35. Gerondopoulos A, Langemeyer L, Liang J-R, Linford A, Barr FA: BLOC-3 mutated in Hermansky-Pudlak syndome is a Rab32/38 guanine nucleotide exchanger factor. Curr Biol 2012, 22(22):2135-2139. 
36. Keefe RS, Harvey PD, Goldberg TE, Gold JM, Walker TM, Kennel C, Hawkins K: Norms and standardization of the Brief Assessment of Cognition in Schizophrenia (BACS). Schizophr Res 2008, 102(1-3):108-115.

37. Kaneda Y, Sumiyoshi T, Keefe R, Ishimoto Y, Numata S, Ohmori T: Brief assessment of cognition in schizophrenia: validation of the Japanese version. Psychiatry Clin Neurosci 2007, 61(6):602-609.

38. American Psychiatric Association: Diagnostic and Statistical Manual of Mental Disorders, Fourth Edition, Text Revision. Washington, DC: American Psychiatric Publishing Inc.; 2000.

39. World Medical Association. [http://www.wma.net]

40. Kay SR, Fiszbein A, Opler LA: The positive and negative syndrome scale (PANSS) for schizophrenia. Schizophr Bull 1987, 13(2):261-276.

41. Ota T, lida J, Sawada M, Suehiro Y, Kishimoto N, Tanaka S, Nagauchi K, Nakanishi Y, Yamamuro K, Negoro H, Iwasaka H, Sadamatsu M, Kishimoto T: Comparison of pervasive developmental disorder and schizophrenia by the Japanese version of the National Adult Reading Test. Int J Psychiatry Clin Pract 2013, 17(1):10-15.

42. Ohnishi T, Hashimoto R, Mori T, Nemoto K, Moriguchi Y, lida H, Noguchi H, Nakabayashi T, Hori H, Ohmori M, Tsukue R, Anami K, Hirabayashi N, Harada S, Arima K, Saitoh O, Kunugi H: The association between the Val158Met polymorphism of the catechol-O-methyl transferase gene and morphological abnormalities of the brain in chronic schizophrenia. Brain 2006, 129(2):399-410

43. Takei K, Yamasue $H$, Abe O, Yamada H, Inoue H, Suga M, Muroi M, Sasaki H, Aok $\mathrm{S}$, Kasai K: Structural disruption of the dorsal cingulum bundle is associated with impaired Stroop performance in patients with schizophrenia. Schizophr Res 2009, 114(1-3):119-127.

44. Crawford JR, Besson JA, Bremner M, Ebmeier KP, Cochrane RH, Kirkwood K: Estimation of premorbid intelligence in schizophrenia. Br J Psychiatry 1992, 161:69-74

45. PLINK: Whole genome data analysis toolset. [http://pngu.mgh.harvard. edu/ purcell/plink/]

46. Purcell S, Neale B, Todd-Brown K, Thomas L, Ferreira MA, Bender D, Maller J, Sklar P, de Bakker PI, Daly MJ, Sham PC: PLINK: a tool set for wholegenome association and population-based linkage analyses. Am J Hum Genet 2007, 81(3):559-575.

47. Haploview. [http://www.broadinstitute.org/scientific-community/science/ programs/medical-and-population-genetics/haploview/haploview]

48. Barrett JC, Fry B, Maller J, Daly MJ: Haploview: analysis and visualization of LD and haplotype maps. Bioinformatics 2005, 21:263-265.

49. Begemann M, Grube S, Papiol S, Malzahn D, Krampe H, Ribbe K, Friedrichs H, Radyushkin KA, El-Kordi A, Benseler F, Hannke K, Sperling S, Schwerdtfeger D, Thanhäuser I, Gerchen MF, Ghorbani M, Gutwinski S, Hilmes C, Leppert R, Ronnenberg A, Sowislo J, Stawicki S, Stödtke M, Szuszies C, Reim K, Riggert J, Eckstein F, Falkai P, Bickeböller H, Nave KA, et al: Modification of cognitive performance in schizophrenia by complexin 2 gene polymorphisms. Arch Gen Psychiatry 2010, 67(9):879-888.

50. Blom G: Statistical Estimates and Transformed Beta Variables. New York, NY: Wiley; 1958.

51. G*Power 3. [http://www.psycho.uni-duesseldorf.de/abteilungen/aap/ gpower3/]

52. Faul $F$, Erdfelder $E$, Lang AG, Buchner A: G*Power 3: a flexible statistical power analysis program for the social, behavioral, and biomedical sciences. Behav Res Methods 2007, 39(2):175-191.

53. Faul F, Erdfelder E, Buchner A, Lang AG: Statistical power analyses using G*Power 3.1: tests for correlation and regression analyses. Behav Res Methods 2009, 41(4):1149-1160.

54. Jurado $M B$, Rosselli $M$ : The elusive nature of executive functions: a review of our current understanding. Neuropsychol Rev 2007, 17(3):213-233.

55. Morris RG, Rushe T, Woodruffe PW, Murray RM: Problem solving in schizophrenia: a specific deficit in planning ability. Schizophr Res 1995 14(3):235-246

56. Sullivan JR, Riccio CA, Castillo CL: Concurrent validity of the tower tasks as measures of executive function in adults: a meta-analysis. Appl Neuropsychol 2009, 16(1):62-75.

57. Dagher A, Owen AM, Boecker H, Brooks DJ: Mapping the network for planning: a correlational PET activation study with the Tower of London task. Brain 1999, 122(10):1973-1987.

58. Zhu $Y$, Liu $X$, Wang $H$, Jiang $T$, Fang $Y$, Hu H, Wang G, Wang $X$, Liu Z, Zhang K: Reduced prefrontal activation during Tower of London in first-episode schizophrenia: a multi-channel near-infrared spectroscopy study. Neurosci Lett 2010, 478(3):136-140.
59. Joyce EM, Hutton SB, Mutsatsa SH, Barnes TR: Cognitive heterogeneity in first-episode schizophrenia. Br J Psychiatry 2005, 187(6):516-522.

60. Haut MW, Kuwabara H, Leach S, Arias RG: Neural activation during performance of number-letter sequencing. App/ Neuropsychol 2000, 7(4):237-242.

61. Prata DP, Mechelli A, Fu CH, Picchioni M, Kane F, Kalidindi S, McDonald C, Howes O, Kravariti E, Demjaha A, Toulopoulou T, Diforti M, Murray RM, Collier DA, McGuire PK: Opposite effects of catechol-O-methyltransferase Val158Met on cortical function in healthy subjects and patients with schizophrenia. Biol Psychiatry 2009, 65(6):473-480.

62. Allen Brain Atlas. [http://www.brain-map.org]

63. Kishi T, Moriwaki M, Kawashima K, Okochi T, Fukuo Y, Kitajima T, Furukawa O, Naitoh H, Fujita K, Iwata N: Investigation of clinical factors influencing cognitive function in Japanese schizophrenia. Neurosci Res 2010, 66(4):340-344.

64. Ogino S, Miyamoto S, Tenjin T, Kitajima R, Ojima K, Miyake N, Funamoto Y, Arai J, Tsukahara S, Ito Y, Tadokoro M, Anai K, Tatsunami S, Kubota H, Kaneda Y, Yamaguchi N: Effects of discontinuation of long-term biperiden use on cognitive function and quality of life in schizophrenia. Prog Neuropsychopharmacol Biol Psychiatry 2011, 35(1):78-83.

65. Fatjó-Vilas M, Papiol S, Estrada G, Bombín I, Peralta V, Rosa A, Parellada M, Miret S, Martín M, Lázaro L, Campanera S, Muñoz MJ, Lera-Miguel S, Arias B, Navarro ME, Castro-Fornieles J, Cuesta MJ, Arango C, Fañanás L: Dysbindin1 gene contributes differentially to early- and adult-onset forms of functional psychosis. Am J Med Genet Part B 2011, 156B(3):322-333.

66. Donohoe G, Morris DW, Clarke S, McGhee KA, Schwaiger S, Nangle JM, Garavan $\mathrm{H}$, Robertson $\mathrm{H}$, Gill M, Corvin A: Variance in neurocognitive performance is associated with dysbindin-1 in schizophrenia: a preliminary study. Neuropsychologia 2007, 45(2):454-458.

doi:10.1186/1471-244X-13-276

Cite this article as: Kuratomi et al:: Association of the Hermansky-Pudlak syndrome type 4 (HPS4) gene variants with cognitive function in patients with schizophrenia and healthy subjects. BMC Psychiatry 2013 13:276.

\section{Submit your next manuscript to BioMed Central and take full advantage of:}

- Convenient online submission

- Thorough peer review

- No space constraints or color figure charges

- Immediate publication on acceptance

- Inclusion in PubMed, CAS, Scopus and Google Scholar

- Research which is freely available for redistribution

Submit your manuscript at www.biomedcentral.com/submit
C) Biomed Central 